\title{
Longitudinal relations among inattention, working memory, and academic achievement: Testing mediation and the moderating role of gender
}

Sarah Gray, Maria Rogers, Rhonda Martinussen, Rosemary Tannock

Introduction: Behavioral inattention, working memory (WM), and academic achievement share significant variance, but the direction of relationships across development is unknown. The aim of the present study was to determine whether WM mediates the pathway between inattentive behaviour and subsequent academic outcomes. Methods: 204 students from grades 1-4 (49.5\% female) were recruited from elementary schools. Participants received assessments of WM and achievement at baseline and one year later. WM measures included a visual-spatial storage task and auditory-verbal storage and manipulation tasks. Teachers completed the SWAN behaviour rating scale both years. Mediation analysis with PROCESS (Hayes, 2013) was used to determine mediation pathways. Results: Teacher-rated inattention indirectly influenced math addition fluency, subtraction fluency and calculation scores through its effect on visual-spatial WM, only for boys. There was a direct relationship between inattention and math outcomes one year later for girls and boys. Children who displayed better attention had higher WM scores, and children with higher WM scores had stronger scores on math outcomes. Bias-corrected bootstrap confidence intervals for the indirect effects were entirely below zero for boys, for the three math outcomes. WM did not mediate the direct relationship between inattention and reading scores. Discussion: Findings identify inattention and WM as longitudinal predictors for math addition and subtraction fluency and math calculation outcomes one year later, with visual-spatial WM as a significant mediator for boys. Results highlight the close relationship between inattention and WM and their importance in the development of math skills. 
9 Sarah A. Gray, M.A. Sa.gray@mail.utoronto.ca. Ontario Institute for Studies in Education, University 10 of Toronto.

11

Maria Rogers, Ph.D. Maria.Rogers@uottawa.ca. University of Ottawa.

14 Rhonda Martinussen, Ph.D. Rhonda.martinussen@utoronto.ca. Ontario Institute for Studies in 15 Education, University of Toronto.

16

17 Rosemary Tannock, Ph.D. Rosemary.tannock@utoronto.ca. Ontario Institute for Studies in Education, 18 University of Toronto; Research Institute of the Hospital for Sick Children.

19

20

21

22

23

24

25

26

27

28

29

30 
Longitudinal relations among inattention, working memory, and academic achievement: Testing mediation and the moderating role of gender.

A strong body of literature has provided evidence of a link between inattentive behavior in the

classroom and academic underachievement (for a review, see Polderman, Boomsma, Bartels, Verhulst,

36 \& Huizink, 2010). As a behavioural descriptor, attention refers to overt on-task behaviours (for example, visual fixation on a relevant stimulus such as a teacher) as well as organization (for example, keeping track of materials) and is measured using behavioural rating scales (for example, the SWAN rating scale, Conners-3) filled out by a parent or teacher who regularly observes a child's behaviour. Inattention refers to off-task behavior, and includes the concept of disorganization. Although

41 Polderman and colleagues included the behavioural dimension of hyperactivity in their review, it is the 42 dimension of inattention that has consistently been found to be a risk factor for poor academic 43 achievement across development (Garner et al., 2014; Pingault et al., 2011). Teacher-ratings of

44 behavioural inattention are more strongly linked to academic outcomes than are parent-ratings, and are more sensitive to the demands of the classroom environment (Garner et al., 2014).

Inattention and math achievement. Teacher-rated inattention is an independent predictor of performance in multiple achievement domains that are important throughout the elementary school 48 years, including arithmetic fluency (Fuchs et al., 2006; Lewandowski, Lovett, Parolin, Gordon, \& 49 Codding, 2007), arithmetic word problems (Fuchs et al., 2006; Swanson, 2011) and algorithmic 50 computation (Fuchs et al., 2006; Li \& Geary, 2013; Raghubar et al., 2009), as well as for composites of 51 arithmetic fluency and algorithmic computation (Fitzpatrick \& Pagani, 2013; Gold et al., 2013). These 52 three domains of math are distinguishable (Fuchs et al., 2006). Arithmetic fluency is defined as solving 53 simple math facts, with a timing component, where students are expected to quickly and accurately 54 solve math fact problems. As children become efficient counters, associations between pairs of 
55 numbers become consolidated in long-term memory, therefore relying more on retrieval memory and

56 putting less burden on working memory (WM) for answering math fact questions fluently (Geary,

57 Brown, \& Samaranayake, 1991). Petrill and colleagues (2012) found that arithmetic fluency is

58 genetically distinct from other non-timed measures of math calculation, problem solving and number

59 concepts (Petrill et al., 2012). Arithmetic fluency plays a role in the development of algorithmic

60 computation, which is defined by Fuchs et al. (2006 p.30) as "adding, subtracting, multiplying or

61 dividing whole numbers, decimals or factions using algorithms and arithmetic." This type of math is

62 differentiated from arithmetic fluency as it includes carrying and borrowing; moving outside of simple

63 arithmetic to include the use of algorithm, and necessitating the ability to follow procedural steps as

64 well as reliance on math fact retrieval.

65 Inattention and reading achievement. Reading fluency is another important domain of

66 achievement during the elementary school years, as it is a consistent predictor of later reading

67 comprehension skills (Pearce \& Gayle, 2009; Roehrig, Petscher, Nettles, Hudson, \& Torgesen, 2008).

68 The ability to read fluently in the early grades is also predictive of high-stakes achievement test scores

69 in elementary and middle school, and continues to predict reading comprehension scores into

70 adulthood (Baker et al., 2014; Tighe \& Schatschneider, 2014). There is some evidence that reading

71 fluency is linked to attention, in that inattentive behavior is a predictor of poor reading fluency

72 outcomes in typical developing school children (Pham, 2013). A study using a community sample of

73 elementary school children found that mid-term teacher-rated inattention predicted word reading

74 fluency at the end of the same year, although it did not predict basic reading (word reading without

75 timed component and decoding ability) (Grills-Taquechel, Fletcher, Vaughn, Denton, \& Taylor, 2013).

76 Studies with clinical groups have also found that children with Attention-Deficit/Hyperactivity

77 Disorder (ADHD) have lower reading fluency outcomes than their peers (Jacobson et al., 2011;

78 Willcutt, Pennington, Olson, \& DeFries, 2007). 
The mechanisms of association between inattention and math and reading outcomes are not yet delineated. Although it is teacher-rated inattention that is strongly linked with poor academic

81 achievement, attention encompasses both behavioural and cognitive components, and these two aspects

82 of attention do not readily map onto each other (for a review, see Tannock, 2003). As a cognitive

83 descriptor, attention refers to a complex set of processes that operate through a series of neural

84 networks. Three specific networks have been delineated; alerting, orienting and executive control

85 (Posner \& Rothbart, 2007). It is not known whether cognitive aspects of attention mediate the

86 relationship between behavioural inattention and poor academic achievement. Within the cognitive

87 network of executive control, the functional domain of working memory (WM) has been implicated in

88 math and reading achievement and is strongly related to inattention, and thus presents as a possible

89 mediating variable within this relationship (Fuchs et al., 2005; Martinussen \& Tannock, 2006;

90 Swanson \& Beebe-Frankenberger, 2004).

91 A number of educational studies draw upon Baddeley's multicomponent model of WM in

92 which WM is viewed as a limited-capacity system that temporarily holds and manipulates information.

93 This model includes separate storage modules for auditory-verbal (phonological loop) and visual-

94 spatial information (visual-spatial sketchpad), and a central executive component that interfaces with

95 other systems such as long term memory and perceptual systems (Baddeley, 2003, 2012). Within this

96 model, both short-term storage modules and modules that process or manipulate information are

97 considered to be part of WM. Following from this model, as well as research that provides evidence

98 that these domains overlap significantly and may not tap into separate constructs, both storage and

99 manipulation components will be conceptualized under the domain of WM within the current study

100 (Colom, Shih, Flores-Mendoza, \& Quiroga, 2006; Engle, Tuholski, Laughlin, \& Conway, 1999;

101 Miyake, Friedman, Rettinger, Shah, \& Hegarty, 2001). Differences have been found, however, 
102 between tasks that require short-term storage or manipulation, with the latter showing relationships to 103 fluid intelligence and cognitive aptitudes (Cowan, 2008; Engle et al., 1999).

104 Children with poor WM ability demonstrate impaired academic performance, including 105 impaired performance on tests of overall reading and math, and reading fluency (Alloway, Gathercole, 106 Kirkwood, \& Elliott, 2009; Bental \& Tirosh, 2007; Gathercole \& Pickering, 2000; Jacobson et al., 107 2011). These same children are rated by teachers as having more problems with inattention and 108 distractibility (Alloway et al., 2009). Similarly, a study with a sample of children representing the 109 normal range of WM ability found that WM is long-term predictor of literacy and numeracy outcomes 110 (Alloway, Elliott, \& Place, 2010). A recent study found that WM (a composite of both auditory-verbal 111 and visual-spatial WM), was an important predictor of math achievement for students with high levels 112 of ADHD symptoms (Rennie, Beebe-Frankenberger, \& Swanson, 2014). Visual-spatial storage, when 113 measured in pre-school children, was also found to predict first grade math outcomes (Bull, Espy, \& 114 Wiebe, 2008). Working memory deficits often co-occur with attention difficulties, both in those 115 individuals with disorders of attention and across the spectrum of typical behaviour (Gathercole \& 116 Pickering, 2000; Martinussen \& Tannock, 2006; Willcutt, Doyle, Nigg, Faraone, \& Pennington, 2005).

117 Moreover, when examined across one school year, inattention, WM and academic fluency were found 118 to share a significant amount of variance in a community sample of elementary school children (Gray, 119 Rogers, Martinussen, \& Tannock, submitted manuscript), supporting the hypothesis that these three 120 factors comprise a triad of impairment during the elementary years and into high-school (Martinussen, 121 Hayden, Hogg-Johnson, \& Tannock, 2005; Rogers, Hwang, Toplak, Weiss, \& Tannock, 2011). Currently there is no robust evidence regarding the direction of the relationships within this

123 triad of impairment, and causal pathways are unknown. One study found that trajectories of ADHD 124 behaviour could be established based on cognitive features at 15 and 24 months, and that those with 125 more severe ADHD symptoms in grade 3 did show some behavioural differences prior to starting 
126 school (Arnett, Macdonald, \& Pennington, 2013). The researchers found that early signs of both

127 behavioural and cognitive difficulties were associated with a stable trajectory of poor academic

128 achievement into grade 3 (Arnett, Macdonald, \& Pennington, 2013). Although this study provides

129 evidence as to the early emergence of both behavioural and cognitive difficulties, and their association

130 with low academic achievement, a grouping of cognitive features based on general intelligence and

131 grouping inattention with an 'externalizing behaviour' composite does not allow for looking at

132 domains of specific relevance to academic achievement, such as WM and the spectrum of inattention.

133 Another study, examining a sample of term and pre-term children, found that a measure of executive

134 function (EF) (including visual-spatial WM) did not contribute unique variance to teacher-rated

135 inattention scores in preschool, but visual-spatial span did contribute unique variance to these scores in

136 primary school (Aarnoudse-Moens, Weisglas-Kuperus, Duivenvoorden, van Goudoever, \& Oosterlaan,

137 2013). These studies indicate changes in the relationship between teacher-rated inattention and WM

138 throughout early development.

139 Other studies have investigated possible mediators that provide some account of the consistent

140 relationship between inattention and academic achievement. In a sample of high school students

141 presenting with clinical and sub-clinical levels of ADHD symptoms, WM was found to be a mediator

142 of the relationship between inattention and reading and math composite scores (Rogers et al., 2011).

143 Thorell (2007) examined WM in a mediating role within an EF composite score. They found that this

144 EF score mediated the relationship between inattention and pre-academic skills in kindergarten-aged

145 children (Thorell, 2007).

146 The current study sought to extend these studies to a community sample of elementary school

147 children and to further delineate the nature of the relationship between classroom inattention, WM

148 domains and academic achievement through using a longitudinal mediation design. Differential

149 influences of visual-spatial and auditory-verbal WM are of interest, given previous research that 
150 implicates visual-spatial WM as an important factor in math achievement in elementary and high

151 school, and previous findings of differential relationships between WM domain and achievement

152 domain (Li \& Geary, 2013; Rogers et al., 2011). As sex differences are often evident in overall levels

153 of inattentive behavior (Gershon, 2002), sex was investigated in the current study. Moreover, given

154 recent, although limited evidence that sex differences in attention disorders may be due to underlying

155 genetic and cognitive differences between the sexes (Arnett, Pennington, Willcutt, DeFries, \& Olson,

156 2014), we included sex as a moderator of the direct and indirect effects. Based on the previous studies

157 described, as well as on examination of this sample within a one year time frame (Gray, Rogers,

158 Martinussen, \& Tannock, submitted manuscript), it is hypothesized that there will be a direct

159 relationship between classroom inattention at one point in time and both math and reading outcomes

160 one year later. Additional hypotheses posit that inattention will indirectly influence math outcomes

161 through visual-spatial and auditory-verbal WM, and indirectly influence reading outcomes through

162 auditory-verbal WM.

163

164

165

166

167

168

169

170

171

172

\section{Materials and Methods}

\section{Participants}

Participants were 204 elementary school-aged children (49.5\% female) in grades 1-4 (ages 5-9, $\mathrm{M}=7.67, \mathrm{SD}=0.91$ ), who were drawn from a larger sample of 524 students, as described below.

Students and their teachers and parents were recruited from a large suburban and rural school district in Southern Ontario, Canada. The 7 participating schools ( $20 \%$ of the 33 schools in the district) were stratified across socio-economic groups. Stratifying for sex, this subsample of 204 was created by taking 2-3 students in each class from the highest, middle and lowest ranking levels of attention, based on teacher ratings of inattentive behaviour in the classroom, which were rank ordered. This smaller 
173 sample, representative of the continuum of attention across students, was then given more in-depth

174 academic and cognitive assessments in the second half of each study year.

175 The majority of participants were Caucasian (80.6\%) with English as their primary language

176 (83.3\%). All students that were in mainstream English or French classrooms (29\% in French

177 Immersion) were eligible for the study, providing that they did not have major sensory or motor

178 impairment that would preclude the ability to complete the tasks or hear instructions. Teacher reports

179 indicated that $11.8 \%$ of sample had an Individual Education Plan (IEP) with 5.5\% identified with

180 ADHD, 3.8\% a learning disability, $4.9 \%$ a language impairment, $1.6 \%$ a behaviour difficulty, $0.5 \%$ a

181 developmental disability. In terms of parent education level, $2.7 \%$ of participating parents had less than

182 high school education, 5.5\% had graduated from high school or an equivalent, $57.7 \%$ had graduated

183 from college or university, and $11 \%$ had a post graduate degree. No sex differences were found on any

184 demographic variables, with one exception: females were more likely to have a parent $(92.3 \%$ of

185 parents who filled out questionnaires were mothers, and 7.7\% were fathers) with less than high school

186 education.

187 Procedures

188 In accordance with procedures approved by the hospital and school board Institutional Review

189 Boards (REB approval number 1000013136), study information was presented in an initial meeting

190 with principals of potential participant schools. Interested principals then contacted the research team,

191 after which an information session for teachers was held at each participating school.

192 In the Canadian school system, the school year starts in September and ends in June. Thus,

193 November is in term one (term A) and April is in term two (term B) of the school year. In the current

194 study, there were four waves of data collection across two years. The first wave took place in

195 November of study Year 1, and will be referred to as Year 1 term A (Year 1A). The second wave took 
196 place in April of Year 1 and will be referred to as Year 1 term B (Year 1B). Similarly, in the second 197 year of the study, data was collected in November (Year 2A) and in April (Year 2B).

198 Teachers and parents who gave written informed consent to participate in the current study 199 completed questionnaire packages in November of Years 1 and 2 of the study (Year 1A, Year 2A).

200 This gave the teachers two months to get to know their students (September and October) before 201 completing the questionnaires. At the time of consent, parents were aware that their children might 202 participate in either two or four testing sessions across the two years. Children who had written 203 informed consent from parents and gave verbal assent, participated in academic testing sessions in 204 November of Years 1 and 2 of the study (Year 1A, Year 2A). All assessments were conducted in 205 English, and all materials were English. As described above, after the teacher-rated inattentive 206 behaviour questionnaires were completed, a subset of students from each class, from the lowest, middle 207 and highest bracket of the continuum of attention were selected to participate in further tests of 208 cognitive (including working memory) and academic functioning. These further tests were 209 administered to the same subset of 204 students in April of study Years 1 and 2 (Year 1B, Year 2B).

210 Measures

211 The following measures, including a behaviour questionnaire, and standardized tests of

212 academic achievement and WM were selected from a larger study that included a range of behavioural, 213 cognitive and academic measures.

214 Assessment of classroom attention. Classroom attention was measured using the Strengths 215 and Weaknesses of Attention-Deficit/Hyperactivity Disorder Symptoms and Normal Behaviour Scale 216 (SWAN; http://www.adhd.net/SWAN_SCALE.pdf), completed by teachers in November of Year 1 and 217 Year 2 (Year 1A, Year 2A) of the study. This scale assesses behaviour using a sensitive 7-point scale $218(3=$ Far below average, $2=$ Below average, $1=$ Slightly below average, $0=$ Average, $-1=$ Slightly 219 above average, -2 = Above average, $-3=$ Far above average). This allows for measuring the full range 
220 of behavioural attention in a population-based sample, using positively worded probes. This design

221 avoids psychometric flaws such as negative or positive skewedness that can arise with 4 point-scales,

222 in which scores for the majority of children cluster around zero. The SWAN scale provides a range of

223 scores for children who have average attention as well as good or poor attention at either end of the

224 spectrum (Arnett et al., 2011). The scale is divided into 'inattention' and 'hyperactivity' subscales. The

225 inattention subscale only was employed in this study, considering the large body of evidence that links

226 inattention with academic achievement, and does not provide evidence of such a link between

227 hyperactivity and academic outcomes (Garner et al., 2014; Rabiner \& Coie, 2000). This inattention

228 subscale consists of 9 items. Internal consistency of this scale is acceptable and consistent with other

229 often used behaviour rating scales (full scale, $\alpha=.88$; inattention subscale, $\alpha=.94$. Test-retest

230 reliability estimates for the full scale range from .72 -.90 (Arnett et al., 2011; Swanson et al., 2001;

231 Young, Levy, Martin, \& Hay, 2009). In the current sample, a correlation of .74 was found for the

232 inattention subscale at Year 1A and Year 2A. Scores are distributed and coded based on a 7-point

233 scale: Negative scores indicate stronger attention; lower levels of inattentive behaviour, while positive

234 scores indicate weak attention; higher levels of inattentive behaviour.

235 Measures of math achievement. To assess students' math abilities across the two years,

236 subtests from two commonly used batteries were administered at each wave of data collection. The

237 addition and subtraction probes from AIMSweb ${ }^{\circledR}$ M-CBM, Mathematics Curriculum-Based

238 Measurement was used to test grade-level fluency in addition and subtraction, therefore was used as a

239 measure of arithmetic fluency. This reliable and valid curriculum-based measure (CBM) assesses math

240 fluency; probes are taken from the school curriculum and standardized. Test-retest reliability is high

241 (.87), as is inter-rater reliability (.83), and alternate form reliability is moderate (.66) (Thurber, Shinn,

$242 \&$ Smolkowski, 2002). Forms for grades 1-3 included 60 math fact problems (basic subtraction and

243 addition), and forms for grade 4 students included 84 math fact problems. Math problems did not 
244 require borrowing or carrying, and contained digits $0-12$, thus some computations were either single (1 $245+8)$ multi-digit $(11-8)$. The content was the same for both forms, with the only difference being the 246 number of available questions. The scoring is unique in that credit is given to each individual correct 247 digit that appears in the solution. This allows for a more precise analysis of a child's math skills, as it 248 captures emerging and partial skills as well as fully mastered skills, thus providing a sensitive measure

249 of math fluency. The test is administered in a group format, and students are given 2 minutes to 250 complete as many problems as they can. This task is sensitive to both short-term and long-term 251 improvement in student achievement, thus is appropriate for a longitudinal study design (Thurber et al., 252 2002).

The Math Calculation subtest from the Woodcock-Johnson - III Tests of Achievement (WJ-

254 IIIACH) was administered to assess a second component of math achievement, within the area of 255 algorithmic computation. The WJ-IIIACH is a highly reliable standardized battery that can be used 256 throughout the academic trajectory. Internal consistency reliability is .86 for Math Calculation 257 (Woodcock, McGrew, \& Mather, 2001a). The problems in this task do start out with simple arithmetic 258 (i.e. $2+3$ ), similar to the CBM addition and subtraction fluency tasks, however problems quickly 259 move into borrowing and carrying (i.e. $16+6$ ) and to multiplication and division. The difficulty of the 260 questions increases as the student progresses. The test is timed, however time is not emphasized in the 261 instructions and a full seven minutes is given for the participants to complete as many questions as they 262 can. Partial points are not given as in the CBM tasks, the questions in this task are either given one 263 point or zero points. 
Previous research has found that addition and subtraction fluency (short time limit for simple arithmetic problems) and math calculation (algorithmic computation, longer time limit), cluster together under the narrow math ability factor, and both are related to perceptual speed. However, these domains of math are separable factors, and only math fluency is related to the broad ability of processing speed (Woodcock et al., 2001a).

Measures of reading achievement. Reading fluency was assessed using the Dynamic loud, and are instructed to read as accurately as possible, and to read as many words as they can within one minute. Points are deducted for omissions, substitutions, inaccurate pronunciation and hesitations over 3 seconds. The median number of errors across the three passages is scored, as is the median number of correct words; this latter score was used as the oral reading fluency measure in the current study. One subtest from the Woodcock-Johnson - III Tests of Achievement (WJ-IIIACH), Letter-Word Identification, was used from the "Reading ability" cluster of this battery in order to test fluent single word reading ability. This subtest presents single words listed on a page and words increase in difficulty as the student progresses. Credit is given if the word is said out loud smoothly and accurately. Throughout this paper, the CBM Oral Reading Fluency subtest will be referred to as 'Reading Fluency' and the WJ-ACHIII Letter-Word-Identification test will be referred to as 'Word Reading.' strong psychometric properties and in order to extend previous findings from studies using these measures. The Wechsler Intelligence Scale for Children (WISC-IV), Digit Span Subtests (DS) is a 
292.82 (Wechsler, 2003). The test requires participants to listen to and recall a series of digits. In the Digit

293 Span Forward task, participants are asked to recall the digits exactly as heard, while in the Digit Span

294 Backward task, participants are asked reproduce the digits heard in backward sequence. The

295 standardized composite score of these two tasks was used in the current study. The Wide Range

296 Assessment of Memory and Learning (WRAML-2), Finger Windows Forward Subtest (FWF) was

297 administered in order to assess visual-spatial WM. This test has high internal consistency (.99) and taps

298 into the visual-spatial storage component of WM (Sheslow \& Adams, 2003). This battery does not

299 contain a visual-spatial WM task that requires manipulation in addition to short-term storage.

300 Participants are presented with an 8x11 plastic grid with 'windows' distributed throughout the grid.

301 Participants, who are seated directly across from the examiner, are asked to replicate the examiner's

302 visual sequence, created with a pencil tapping different sequences of 'windows.' The sequence

303 becomes longer as participants progress. A standardized score is calculated from the total number of

304 correct sequences that the participant is able to replicate.

305 Statistical Approach

306 Missing data was imputed according to the methods suggested by McKnight, McKnight, Sidani,

307 \& Figueredo (2007), when not more than $10-15 \%$ of data is missing. It should be noted that for

308 analyses in which parental education is a covariate, only 182 records were complete with this

309 information, thus analyses are based on this reduced sample size. No significant outliers were detected.

310 Assumptions of normality and homoscedasticity were satisfied, with the exception of the Year 2B

311 Math Addition variable, where the Levene's test was significant for the male sample (however, the test

312 was not significant for the full sample). As a precaution, the HC3 test in the SPSS macro PROCESS

313 (Preacher \& Hayes, 2008) was used to produce heteroscedasticity-consistent standard error estimates

314 for this variable. 
Relationships between study variables. Partial correlations were calculated to examine the

316 relationship between all study variables. Age was placed as a control variable, as it is an important

317 factor in CBMs across grades and initial analysis indicated that age was differentially related to WM

318 variables. A one-way ANOVA was used to examine sex differences between study variables.

319 Mediation analyses. All mediation models were designed with visual-spatial WM and

320 auditory-verbal WM at Year 1B as parallel mediators between teacher-rated inattention at Year 1A and

321 academic outcomes at Year 2B, with sex as a moderator. Moderated mediation analyses were carried

322 out using the PROCESS macro for SPSS, developed and described by Hayes (2013) and Preacher \&

323 Hayes (2008). Their suggested procedures allow for detecting the difference between the direct effect

324 of a predictor on an outcome variable, and the indirect effect after accounting for the mediator. Using

325 this macro also allows for testing the relative strength of auditory-verbal WM and visual-spatial WM as

326 mediators within each analysis (Hayes, 2013; Preacher \& Hayes, 2008). Year 1A academic scores were

327 added in each analysis as a covariate. This model allows for partialling out the influence of baseline

328 academic scores collected at Year 1A and examining influences of each variable across time. Outcome

329 variables were examined in separate models instead of in one simultaneous model in order to elucidate

330 the role of inattention and WM in the development of specific skills within math and reading at the

331 elementary school level. All analyses were carried out with IBM SPSS version 21.

\section{Results}

\section{Correlations between study variables}

Partial correlations between all study variables, controlling for age, are presented in Table 1.

335 Teacher-rated inattention, measured at Year 1A, was significantly correlated in the expected direction

336 with WM measures at Year 1B and all academic outcome variables at Year 2B. All main study

337 variables were significantly correlated in the expected direction at the .01 level, with the exception of

338 visual-spatial and auditory-verbal WM which were not significantly correlated. This finding is 
somewhat unexpected as a previous study using an adolescent sample found that both domains of WM were moderately correlated (Rogers et al., 2011). This finding is discussed further below.

A very strong positive relationship was found between reading fluency and word reading, and between the math addition and subtraction fluency tests, as would be expected given previous research looking at the overlap and differences within these domains of reading achievement (Woodcock et al., 2001), and because the math CBM subtests are both measures of math fact fluency within the same test format. The correlation between math calculation and fluency of math addition and subtraction was lower by comparison, but still strong. This is also expected, as these domains clearly require a similar skill base, however, math fluency is uniquely related to processing speed, while the calculation subtest is related to perceptual speed, and requires higher level procedural skill (Woodcock et al., 2001). Parent education was weakly to moderately correlated with all variables, with the exception of visualspatial WM, which appears to be related to sex but not to parental education in this sample. Conversely, auditory-verbal WM was not related to sex, but was weakly correlated with parental education. Sex was significantly correlated with inattention at the .01 level, and with reading fluency, math subtraction and visual-spatial WM at the .05 level. No significant differences on any study variables were found between students in French Immersion or English classrooms $(p s>.05)$. For all models, parent education and age were entered into the model as covariates along with Y1A academic scores.

[INSERT Table 1. Partial Correlations, Controlling for Age, Between Study Variables for Full Sample $(\mathrm{N}=204)]$ variables, for boys and girls are presented in Table 2. Significant sex differences were found for teacher-rated inattention (boys are rated as more inattentive than girls), reading fluency (girls have 
363 higher scores than boys), and math subtraction (boys have higher scores than girls). However, when

364 applying a Bonferroni family-wise correction for each of the comparisons, with the threshold for

365 significance at an alpha level of .0063, the only remaining sex difference was between teacher-rated

366 inattention. The eta-squared for this significant difference is 0.13 , therefore $13 \%$ of the variance in

367 teacher-rated inattention is accounted for by sex. To assess sex differences in the hypothesized model,

368 sex was entered as a moderator of the mediation analyses.

[INSERT Table 2. Means and Standard Deviations for Study Variables, for Girls and Boys]

\section{Mediation analyses}

Math Outcomes. Moderated mediation analyses with two parallel mediators, and sex as a

moderator, conducted using ordinary least squares (OLS) path analysis, revealed that teacher-rated inattention indirectly influenced math addition and subtraction fluency outcomes through its effect on visual-spatial WM (see Figures 1 and 2), but for boys only. Unstandardized regression coefficients are reported below and in the figures, in accordance with the recommendation of Hayes (2013).

378 Standardized regression coefficients are not produced by PROCESS, and the absolute size of the direct and indirect effects does not indicate whether effects are small or large, as they are tied to our measures that differ across questionnaire/test. A discussion of effect size follows the presentation of results.

Figure 1 presents results of the analysis with addition fluency as the outcome measure. Children who displayed lower levels of teacher-rated inattention at Year 1A (negative scores correspond to better attention) had higher visual-spatial WM scores $(a=-0.16, p<.01)$ at Year 1B, and children with higher visual-spatial WM scores had stronger scores on addition fluency outcomes at Year 2B (visualspatial WM: $b=0.59, p<.05)$. A bias-corrected bootstrap confidence interval (BCa CI) for the 
zero $(-0.13,-0.01)$, therefore is significant. There was also evidence that teacher-rated inattention influenced addition fluency scores the following year independently of its effect on WM (addition $c^{\prime}=$ $-0.28, p<.001)$. The overall model accounts for $59 \%$ of the variance for math addition scores at Year $2\left(\mathrm{R}^{2}=0.59, p<.001\right)$. For boys, WM and teacher-rated inattention account for an additional $4.5 \%$ of the variance in Year 2B addition scores significantly over and above Year 1A addition scores, parent education and age.

INSERT Figure 1. Significant moderated mediation: visual-spatial WM as a mediator of the relationship between teacher-rated inattention and boys' math addition scores one year later.]

Results were similar for subtraction fluency outcomes (see Figure 2). The only difference was that children with higher visual-spatial and auditory-verbal scores had stronger math subtraction outcomes at the end of Year 2B (visual-spatial WM: $b=0.52, p<.05$, auditory-verbal WM: $b=0.59, p$ $<.05)$. The overall model accounts $55 \%$ of the variance for math subtraction scores at Year $2\left(\mathrm{R}^{2}=\right.$ $0.55, p<.001)$. For boys' subtraction scores, $11.4 \%$ of the variance is accounted for by WM and teacher-rated inattention, which is substantially larger than variance accounted for when looking at addition fluency outcomes.

[INSERT Figure 2. Significant moderated mediation: visual-spatial WM as a mediator of the relationship between teacher-rated inattention and boys' math subtraction scores one year later.]

(1) The proposed model was also significant for boys' math calculation outcomes at Year 2B. The conditional indirect effect was significant, $b=-0.03$, BCa CI $[-0.07-0.00]$, as was the direct effect, $b=$ $-0.14, \mathrm{BCa}$ CI $[-0.22,-0.06]$, see Figure 3 . Overall, this model accounted for $53 \%$ of the variance for 
411 Year 2B math calculation scores $\left(\mathrm{R}^{2}=0.53, p<.001\right)$, with teacher-rated inattention and WM

412 accounting for an extra $10.5 \%$ of the variance over and above Year $1 \mathrm{~A}$ calculation scores, parent

413 education and age.

[INSERT Figure 3. Significant moderated mediation: Visual-spatial WM as a mediator of the relationship between teacher-rated inattention and boys' math calculation scores one year later.]

The BCa CIs for all three models passed through zero for girls, thus the mediation through WM was not significant for girls' addition fluency outcomes (visual-spatial WM: [-0.07, 0.01]; auditoryverbal WM: [-0.05, 0.02]), subtraction fluency outcomes (visual-spatial WM: [-0.06, 0.01]; auditoryverbal WM: [-0.07, 0.00]) or math calculation outcomes (visual-spatial WM: [-0.04, 0.01]; auditoryverbal WM: $[-0.04,0.00])$.

A study with a comparable design found that with an adolescent sample, the full model accounted for $40 \%$ of the variance in math outcomes (Rogers et al., 2011), which is somewhat less but still comparable to the variance accounted for in our elementary school-aged sample $(59 \%, 55 \%$ and options for calculating overall effect size are limited to simple mediation models without covariates

428 (Preacher \& Kelley, 2011). Preacher and Kelley outline the difficulty with classic effect size measures, 429 as they do not fit with indirect effects; the product of two regression coefficients. The most robust 430 effect size measure for indirect effects to date is Preacher \& Kelley’s Kappa-squared (K²; Hayes, 2013; 431 Preacher \& Kelley, 2011). Therefore, recognizing that this is of limited applicability to our full model 432 that includes covariates, we calculated the $\mathrm{K}^{2}$ for each significant model, that is, for boys, using visual433 spatial WM at Year 1B as a mediator, teacher-rated inattention at Year 1A as the predictor, and math 434 scores at Year 2B as outcome variables. Results indicate that for math addition, $\mathrm{K}^{2}=.06, \mathrm{BCa} \mathrm{CI}[0.01$, 
$4350.14]$, for math subtraction $\mathrm{K}^{2}=.04$, BCa CI [0.00, 0.13], and for math calculation $\mathrm{K}^{2}=.13$, BCa CI

$436[0.06,0.21]$. This can be interpreted as the indirect effect being about $13 \%$ of the maximum value that

437 it could have been for calculation outcomes, which is between a medium and large effect (Field, 2013;

438 Preacher \& Kelley, 2011). According to Field (2013), this is a 'reasonable' size for psychological

439 science. The effect sizes for the models with math addition and subtraction fluency as outcome

440 variables are between small and medium effects, with the indirect effects being $4 \%$ and $6 \%$ of the

441 maximum value that could have been accounted for in the model.

442 Reading outcomes. When predicting reading outcomes, although approaching significance,

443 there was no significant direct relationship between teacher-rated inattention and reading fluency $(b=$ -

$444.27, p=.055)$ or word reading $(b=-0.02, p=.54)$ scores one year later (see Supplemental Figures 1

445 and 2). There were also no significant mediation effects for reading fluency (visual-spatial WM BCa

446 CI [-0.13, 0.06], auditory-verbal WM BCa CI [-0.16, 0.04]) or word reading (visual-spatial WM BCa

447 CI [-0.02, 0.04], auditory-verbal WM BCa CI [-0.00, 0.02]). Significant predictors of reading fluency

448 at Year 2B were auditory-verbal WM at Year 1B $(b=1.63, p<.05)$, parental education $(b=3.10, p<$

$449.01)$, and Year $1 \mathrm{~A}$ reading fluency scores $(b=0.76, p<.001)$. The only significant predictors of word

450 reading were Year $1 \mathrm{~A}$ word reading scores and age $(b=0.78, p<.001, b=1.88, p<.001)$.

To investigate this same model using WM scores at Year 2B, which allows us to control for

452 previous levels of WM at Year 1B (which we cannot do when using Year 1B scores as the mediating

453 variable), we modeled teacher-rated inattention at Year 1A as the independent variable, academic

454 outcomes at Year 2B as outcome variables with WM at Year 2B as a potential mediator. Results

455 replicate the first model in that visual-spatial WM was a significant mediator of the relationship

456 between teacher-rated inattention and math calculation, the confidence interval for the indirect effect

457 for boys was entirely below zero (BCa CI [-0.10, -0.01]). However WM was not a significant mediator

458 for math CBM addition (BCa CI [-0.10, 0.03]) and subtraction (BCa CI [-0.09, 0.01]), reading fluency 
459 (BCa CI $[-0.10,0.12])$ or word reading (BCa CI $[-0.03,0.03])$. Results from this model need be 460 interpreted with caution, as WM at Year 2B was collected at the same time point as the outcome 461 variables, thus this model is subject to issues with reverse causation.

A reverse model was conducted in order to confirm directionality of the predictor and mediating variables. No mediation models were significant when reversing the role of mediator and

464 independent variable, with Year 1B WM modeled as the independent variable, Year 2A teacher-rated attention as the mediating variable, Year 2B academic variables as outcomes, sex as a moderator and age, parental education, Year 1A academic scores, and Year 1A attention scores as covariates. school children. We hypothesized that inattention would directly and indirectly influence math outcomes through auditory-verbal and visual-spatial WM, and influence reading outcomes through auditory-verbal WM. Using OLS regression based mediation analyses, we found support for a model in which children's classroom inattention, as rated by teachers at the beginning of the school year, was indirectly associated with math outcomes one year later through visual-spatial WM, but only for boys. model held for math CBM addition and subtraction fluency scores as well as math calculation outcomes. 
These new findings raise interesting questions about sex differences in the role of visual-spatial

484 WM on different aspects of math skill development, and about the role of auditory-verbal WM across 485 academic outcomes.

One interesting result that came out of initial analyses is that there was no significant correlation between auditory-verbal and visual-spatial WM measures in this sample. Factor-analytic and neuroimaging studies suggest that the two aspects of WM are distinct domains that overlap, thus proposing a domain-distinct model of WM (Alloway \& Alloway, 2013; Alloway, Gathercole, \& Pickering, 2006; Fassbender \& Schweitzer, 2006). One explanation for the lack of correlation could be that our visual-spatial measure taps into storage, while our auditory-verbal measure taps into both storage and processing components of WM; again, related but separable components (Alloway,

493 Gathercole, \& Pickering, 2006). However, this is unlikely be the explanation in our sample, because 494 there is also no significant correlation between the two measures of storage only (visual-spatial storage 495 and auditory-verbal storage, $r=.12, p=.09$ ). Furthermore, a lifespan study provided support that working memory skills are not driven by differences in function (storage versus manipulation) but by

497 domain differences (Alloway \& Alloway, 2013). This finding is further supported by the current study that suggests domain differences in a sample of children, representative of the full range of inattentive behaviours in a classroom setting.

501 the relationship between teacher-rated inattention and both math fluency and math calculation 502 outcomes is consistent with expectations based on current literature. The visual-spatial domain of WM 503 is consistently linked to math outcomes, both when the measure includes manipulation demands and 504 short-term storage only (Alloway \& Passolunghi, 2011; Bull, Espy, \& Wiebe, 2008; Li \& Geary, 2013; 505 Rogers et al., 2011). However, there were differences in the effect size between the two math domains; 506 with successively larger effect sizes for addition fluency, subtraction fluency and calculation, with the 
507 calculation effect size reaching a medium to large effect $\left(\mathrm{K}^{2}=.13\right)$. The addition and subtraction

508 fluency tests and the WJ-IIIACH math calculation test all require basic math fact skill, and the

509 calculation subtest builds from these basic skills to include procedural knowledge and higher

510 processing demands (Fuchs et al., 2006). There are clear differences between these two measures that

511 may account for the differential influence of WM in terms of effect size and variance accounted for by

512 inattention and WM. Taken together, the robust effect size for calculation scores, and the fact that this

513 model remained significant when controlling for earlier WM (Year 1B) scores, but did not remain

514 significant for fluency scores, indicates that across time, WM appears to play a more significant role as

515 a mediator between inattention and higher-level math calculation skills than for math fluency skills. As

516 elementary school children move from using counting based methods for solving math facts, to fluent

517 memory-based retrieval, there is a parallel shift from activation in the fronto-parietal WM systems, to

518 increased hippocampal activation (Qin et al., 2014). Therefore, it appears that demands on WM for

519 solving math facts are lessened across the early school years, whereas for math calculation tasks that

520 require online processing it is hypothesized that WM load remains high (Geary, 1994). It follows to

521 reason that the influences of inattention on higher-level math outcomes, which require more attention

522 to algorithm and less reliance on fluent retrieval, are partially accounted for by visual-spatial WM.

523 Furthermore, our results that differentiate math fluency from higher-level calculation, with similar

524 effects of visual-spatial WM but different magnitude, can be considered in the context of genetic

525 studies which provide evidence that math fluency is a distinct construct from other domains of math

526 (Petrill et al., 2012).

Another unexpected finding was related to sex differences; in that visual-spatial WM was a

528 significant mediator for boys but not for girls. This was not specifically addressed in an initial

529 hypothesis, but rather came out of analyses that demonstrated sex differences, therefore leading to the

530 examination of sex as a moderator. To our knowledge, this is the first study to examine sex differences 
531 on the role of WM in the relationship between teacher-rated inattention and academic outcomes in the

532 form of standardized achievement. Two studies that looked at variance accounted for by inattention

533 and WM in predicting academic outcomes did not report an assessment of sex differences, outside of

534 the equal distribution of males/females between ADHD and non ADHD groups (Rennie et al., 2014;

535 Rogers et al., 2011). The current results suggest that although sex differences were not found on visual-

536 spatial WM, this construct plays an important role as a mediator between classroom inattention and

537 math outcomes for boys. One possible explanation is through a line of research with adults, which

538 suggests that spatial numerical associations may be represented differently between males and females

539 (Bull, Cleland, \& Mitchell, 2012). Bull and colleagues hypothesize that men may rely more on spatial

540 representations of number, thus providing a theory of why visual-spatial WM played a mediating role

541 in boys' math outcomes. The study was not replicated with children, however, a study with $8^{\text {th }}$ grade

542 students found that boys' scores on mental rotation predicted math achievement, but this was not found

543 for girls (Ganley \& Vasilyeva, 2011). This may suggest that in this higher level of $8^{\text {th }}$ grade math, girls

544 rely less on spatial reasoning for math problem solving than do boys. Future research might examine

545 math anxiety as an additional mediator in this model. Studies have found that math anxiety of female-

546 teachers relates to their female students' math performance via endorsement of sex stereotypes of who

547 is good at math (Beilock, Gunderson, Ramirez, \& Levine, 2010). Therefore it could be that for girls,

548 math anxiety may play a role such that lower performance is not due to visual-spatial WM difficulties,

549 as it is for boys.

Another finding related to sex differences is differential scores for boys and girls on our measure of teacher-rated inattention. In our mediation model, the direct relationship between

552 inattention and math outcomes was significant for both boys and girls. However, our results show

553 higher levels of inattention for boys, which is consistent with existing research. It has been widely

554 reported that boys have higher levels of inattention as rated by teachers and parents, at least for those 
555 who fit diagnostic criteria for ADHD (for a review see Gershon, 2002). However conclusions

556 regarding gender differences in symptoms of inattention are somewhat equivocal, depending on sample

557 (Biederman et al., 2005). Of particular relevance to the current study, Ramtekkar and colleagues found

558 a similar pattern to our results, using the SWAN scale in a community sample of children aged 7-12,

559 with girls showing stronger levels of attention than boys (Ramtekkar, Reiersen, Todorov, \& Todd,

560 2010).

In terms of sex differences on WM tasks, the current results are in line with findings in clinical

562 ADHD populations (for example, Castellanos et al., 2000; Rucklidge \& Tannock, 2002). However, the

563 evidence for sex differences in the general population, on visual-spatial WM is mixed. A body of

564 literature provides consistent findings that males outperform females in visual-spatial rotation tasks,

565 which involve short-term storage as well as transformation (Masters \& Sanders, 1993). A study with

566 children found that males outperformed females on an abstract visual-memory task and a memory for

567 location task, while females outperformed males on two verbal tasks (Modesto-Lowe, Yelunina, \&

568 Hanjan, 2011). However, similar to our results, there were no sex differences on the visual-sequential

569 memory task (most similar to the WRAML finger windows task in the current study) or the digit span

570 tasks (Modesto-Lowe et al., 2011). Similarly, a study with high-school students found no sex

571 differences on a visual-spatial storage task, but did find that males performed better than females on

572 visual-spatial WM tasks that required processing (Kaufman, 2007). Therefore, the current study

573 provides evidence that boys and girls perform equally on the WM tasks included in this study, when

574 examining a community sample of elementary-school aged children.

The finding that auditory-verbal WM did not significantly mediate the relationship between

576 teacher-rated inattention and academic outcomes makes sense given the mean age of our participants

577 and the nature of the math outcome measures. Conflicting results in the role of auditory-verbal WM in

578 math outcomes may be due to the use of a math composite score in previous studies, that included 
579 higher-level math and problem-solving skills that are more strongly associated with verbal WM and 580 executive skills (Passolunghi \& Siegel, 2004; Rogers et al., 2011; Swanson, 2011). In addition, 581 although our sample size did not allow for separate mediation analyses within each grade, differences in relative contribution of auditory-verbal WM to math fluency skills between grades were found in the cited studies. Therefore, another possibility is that the children in our sample are young (mean age is 7.67) and may rely mostly on visual-spatial WM to process information at this stage, not having gone through the developmental shift toward relying more on auditory-verbal WM for information processing (Fastenau, Conant, \& Lauer, 1998; Raghubar, Barnes, \& Hecht, 2010). Another consideration is the presentation format of math problems. It is possible the presentation of problems in a vertical format influenced our results (although 5/45 questions in the math calculation task were horizontal), in that this presentation format recruits more visual-spatial WM resources than math problems that are presented in horizontal format (Trbovich \& LeFevre, 2003). A second main hypothesis in the current study was that inattention would indirectly influence word reading and oral reading fluency outcomes, through auditory-verbal WM. We did not find such an indirect effect, and interestingly, the direct effect of teacher-rated inattention at Year 1A on Year 2B reading fluency and word reading scores was also not significant, while controlling for Year 1A reading fluency scores. In the context of other studies in which inattention is a predictor of reading fluency (for example, Pham, 2013), it is important to note that in the current study, inattention and WM were modeled along with covariates, including parental education, age and Year 1A reading fluency scores, which all significantly predicted reading fluency scores at Year 2B. Examination of this sample within a one year time frame indicated a significant relationship between inattention at Year 1A and 600 reading fluency at Year 1A (Gray et al., submitted manuscript). However, contrary to these findings, 601 inattention does not appear to play a significant role in predicting reading scores one year later 602 independent of its association with reading fluency at Year 1A. This highlights the strong stability of 
603 reading fluency across the elementary school grades. However, auditory-verbal WM was significant

604 predictor for reading fluency. Thus, although not found to play the hypothesized mediating role,

605 auditory-verbal WM is positioned as an important factor in the development of reading fluency across

606 the elementary school years. These results are consistent with the findings of Li and Geary (2013), who

607 also found that visual-spatial WM was not a predictor of reading outcomes, but that gains in visual-

608 spatial WM were associated with stronger math scores at the end of elementary school.

609 Although outside the scope of this paper, future studies could seek to account for other

610 mediators within the relationship between inattention and academic outcomes, in addition to the

611 aforementioned construct of math anxiety. The current study focused on WM, however other cognitive

612 functions, such as processing speed and naming speed are important to consider in relation to academic

613 fluency (Fuchs et al., 2008; Jacobson et al., 2011; Martinussen, Grimbos, \& Ferrari, 2014). WM and

614 processing speed are highly related in the early years of development. However, recent findings

615 suggest that these two constructs independently predict academic fluency as children move into the

616 elementary-school aged years (Clark et al., 2014; Jacobson et al., 2011). Therefore, future studies

617 might examine the role of processing speed as well as WM in the relationship between classroom

618 inattention and academic fluency, while also controlling for more proximal indicators such as

619 phonemic awareness. Other more distal mediators, such as teacher instructional supports, and parent

620 factors such as support with homework, may be important to consider as mediating variables in

621 community samples (Daley \& Birchwood, 2010; Langberg et al., 2011).

622 Strengths of this study include the large sample size, as well as the longitudinal design, in

623 which the predictor is collected before the mediating variables, and the outcome variables are collected

624 one year later. Separation of WM domains and academic skill outcome variables allowed for a more

625 specific understanding of these relationships across two school years. 
There are some limitations to consider in the design and interpretation of this study. One

627 limitation includes our narrow measures for WM, as we did not have a visual-spatial WM measure that

628 required processing or manipulation, the WRAML measure only taps into short-term storage. Although

629 measures used in the current study are highly reliable, future studies might employ a variety of WM

630 measures for each domain, providing the ability to create a comprehensive composite for each domain.

631 A previous study found that visual-spatial storage was a strong predictor of math achievement early in

632 the first grade, but by age 8 , a visual-spatial measure that required manipulation also predicted math

633 outcomes (Bull et al., 2008). It could be that the importance of visual-spatial WM was underestimated

634 by not including a measure that would include all components of visual-spatial WM. Indeed, one study

635 found that the manipulation component of visual-spatial WM did contribute additional variance to

636 math outcomes above the contributions of visual-spatial short-term storage (Geary, Hoard, \& Nugent,

637 2012).

638 Another limitation in our study design is that practical considerations limited data collection

639 time points, such that our mediating variable was collected at 2 time points across two years, whereas

640 the outcome variables were collected at 4 time points. It would have been ideal to have baseline WM

641 measures, however, time allotted by the school for each testing session as well as date restrictions did

642 not permit for collecting cognitive measures for the full sample of 524.

643 Strengthening the confidence in outcomes is the fact that when reverse modeling WM and

644 inattention, where WM is placed at the predictor, and inattention as the mediator, WM measures at

645 Year 1 do not predict levels of inattention in Year 2. Our outcomes regarding visual-spatial WM are

646 afforded more confidence, as we were able to run the analysis with WM at Year 2 as the mediating

647 variable, thus accounting for the influence of prior WM scores (at Year 1). This model continued to

648 reach significance for calculation outcomes, however this model is interpreted with caution, as WM

649 measures at Year 2 were collected at the same time point as the academic outcome measures. Therefore, 
650 although further evidence is needed to substantiate the developmental directionality between

651 inattention and WM, these findings add to our knowledge about longitudinal predictors of academic

652 outcomes in elementary school children and further specify the nature of the relationship between

653 inattention, WM and academic outcomes across elementary school, for typically developing children.

\section{Conclusions}

655 Findings extend previous research and confirm and replicate the body of literature that positions

656 behavioral inattention as a robust predictor of later math achievement, further specifying that this

657 relationship is robust for arithmetic fluency and algorithmic computation, in typically developing

658 elementary school children. Further, findings add new information about the role WM plays for boys

659 and girls in the relationship between inattention and math and reading outcomes across two school

660 years. Contrary to results from cross-sectional studies, our findings provide evidence that after

661 controlling for initial reading fluency scores, auditory-verbal WM is a more robust predictor of reading

662 fluency across two school years, as compared to teacher-rated inattention, which did not predict growth

663 in reading fluency beyond the contribution of Year 1A inattention.

664 Although math fluency shares significant variance with inattention and both domains of WM

665 (Gray, Rogers, Martinussen, \& Tannock, submitted manuscript), the current study provides evidence

666 that boys' classroom inattention (as rated by teachers) directly influences their math fact fluency and

667 math calculation scores across time, controlling for age, parental education and math scores at Year 1.

668 Main findings emphasize that for boys, inattention has a direct effect as well as an indirect influence on

669 math fluency and calculation skills through visual-spatial WM in the elementary school grades. 


\section{References}

676 Aarnoudse-Moens, C. S. H., Weisglas-Kuperus, N., Duivenvoorden, H. J., van Goudoever, J. B., \& Very Preterm Children. PloS One, 8(2), e55994. doi:10.1371/journal.pone.0055994

Alloway, T., \& Alloway, R. (2013). Working memory across the lifespan: A cross-sectional approach. Journal of Cognitive Psychology, 25(1), 84-93.

681 Alloway, T. P., Elliott, J., \& Place, M. (2010). Investigating the relationship between attention and 682 683 working memory in clinical and community samples. Child Neuropsychology : A Journal on Normal and Abnormal Development in Childhood and Adolescence, 16, 242-254.

Alloway, T. P., Gathercole, S. E., Kirkwood, H., \& Elliott, J. (2009). The Cognitive and Behavioral doi:10.1080/09297040903559655

Alloway, T. P., Gathercole, S. E., \& Pickering, S. J. (2006). Verbal and visuospatial short-term and working memory in children: are they separable? Child Development, 77(6), 1698-716. doi:10.1111/j.1467-8624.2006.00968.x

Alloway, T. P., \& Passolunghi, M. C. (2011). The relationship between working memory, IQ, and mathematical skills in children. Learning and Individual Differences, 21(1), 133-137. doi:10.1016/j.lindif.2010.09.013 ADHD symptoms prior to school age. Journal of Child Psychology and Psychiatry, 54(12), 128494. doi:10.1111/jcpp.12104 
696 Arnett, A. B., Pennington, B. F., Willcutt, E. G., DeFries, J. C., \& Olson, R. K. (2014). Sex differences in ADHD symptom severity. Journal of Child Psychology and Psychiatry, n/a-n/a.

698 doi:10.1111/jcpp. 12337

699

700

701

702

703

704

705

706

707

708

709

710

711

712

713

714

715

716

717

718

719

Arnett, A., Pennington, B., Friend, A., Willcutt, E., Byrne, B., Samuelsson, S., \& Olson, R. (2011). The SWAN Captures Variance at the Negative and Positive Ends of the ADHD Symptom Dimension. Journal of Attention Disorders, 17(2), 152-162. doi:10.1177/1087054711427399

Baddeley, A. (2003). Working memory: looking back and looking forward. Nature Reviews. Neuroscience, 4(October), 829-839. doi:10.1038/nrn1201

Baddeley, A. (2012). Working memory: theories, models, and controversies. Annual Review of Psychology, 63, 1-29. doi:10.1146/annurev-psych-120710-100422

Baker, D., Biancarosa, G., Park, B. J., Bousselot, T., Smith, J., Baker, S. K., .., \& Tindal, G. (2014). Validity of cbm measures of oral reading fluency and reading comprehension on high-stakes reading assessments in grades 7 and 8. Reading and Writing, 1-48.

Beilock, S. L., Gunderson, E. a, Ramirez, G., \& Levine, S. C. (2010). Female teachers' math anxiety affects girls' math achievement. Proceedings of the National Academy of Sciences of the United States of America, 107(1), 1860-1863. doi:10.1073/pnas.0910967107

Bental, B., \& Tirosh, E. (2007). The relationship between attention, executive functions and reading domain abilities in attention deficit hyperactivity disorder and reading disorder: a comparative study. Journal of Child Psychology and Psychiatry, 48(5), 455-63. doi:10.1111/j.14697610.2006.01710.x

Biederman, J., Kwon, A., Aleardi, M., Chouinard, V.-A., Marino, T., Cole, H., ... Faraone, S. V. (2005). Absence of gender effects on attention deficit hyperactivity disorder: findings in nonreferred subjects. The American Journal of Psychiatry, 162(June), 1083-1089. doi:10.1176/appi.ajp.162.6.1083 
720 Bull, R., Cleland, A. a., \& Mitchell, T. (2012). Sex Differences in the Spatial Representation of $721 \quad$ Number. Journal of Experimental Psychology: General, 142(1), 181-192. doi:10.1037/a0028387

722 Bull, R., Espy, K. A., \& Wiebe, S. a. (2008). Short-term memory, working memory, and executive 723 functioning in preschoolers: longitudinal predictors of mathematical achievement at age 7 years. 724 Developmental Neuropsychology, 33(3), 205-28. doi:10.1080/87565640801982312

725 Castellanos, F. X., Marvasti, F. F., Ducharme, J. L., Walter, J. M., Israel, M. E., Krain, a, ... Hommer, 726 727 728 D. W. (2000). Executive function oculomotor tasks in girls with ADHD. Journal of the American Academy of Child and Adolescent Psychiatry, 39(5), 644-650. doi:10.1097/00004583200005000-00019

Clark, C. a C., Nelson, J. M., Garza, J., Sheffield, T. D., Wiebe, S. a, \& Espy, K. A. (2014). Gaining control: changing relations between executive control and processing speed and their relevance for mathematics achievement over course of the preschool period. Frontiers in Psychology, 5(February), 107. doi:10.3389/fpsyg.2014.00107

Colom, R., Shih, P. C., Flores-Mendoza, C., \& Quiroga, M. A. (2006). The real relationship between short-term memory and working memory. Memory (Hove, England), 14(February 2015), 804813. doi:10.1080/09658210600680020

Cowan, N. (2008). What are the differences between long-term, short-term, and working memory? Progress in Brain Research, 169(07), 323-338. doi:10.1016/S0079-6123(07)00020-9

Daley, D., \& Birchwood, J. (2010). ADHD and academic performance: why does ADHD impact on academic performance and what can be done to support ADHD children in the classroom? Child: Care, Health and Development, 36(4), 455-64. doi:10.1111/j.1365-2214.2009.01046.x

Engle, R. W., Tuholski, S. W., Laughlin, J. E., \& Conway, a R. (1999). Working memory, short-term memory, and general fluid intelligence: a latent-variable approach. Journal of Experimental Psychology. General, 128(3), 309-331. doi:10.1037/0096-3445.128.3.309 
744 Fassbender, C., \& Schweitzer, J. B. (2006). Is there evidence for neural compensation in attention 745 deficit hyperactivity disorder? A review of the functional neuroimaging literature. Clinical 746 Psychology Review, 26(4), 445-65. doi:10.1016/j.cpr.2006.01.003

747 Fastenau, P., Conant, L., \& Lauer, R. (1998). Working memory in young children: Evidence for 748 modality-specificity and implications for cerebral reorganization in early childhood. Neuropsychologia, 36(7), 643-652.

750 Field, A. (2013). Discovering Statistics using IBM SPSS Statistics. (M. Carmichael, Ed.) (4th ed.). 751 London: SAGE publications.

752 Fitzpatrick, C., \& Pagani, L. S. (2013). Task-oriented kindergarten behavior pays off in later childhood. Journal of Developmental and Behavioral Pediatrics, 34(2), 94-101. doi:10.1097/DBP.0b013e31827a3779

Fuchs, L., Fuchs, D., Hamlett, C., Lambert, W., Stuebing, K., \& Fletcher, J. (2008). Problem Solving and Computational Skill: Are They Shared or Distinct Aspects of Mathematical Cognition? Journal of Educational Psychology, 100(1), 30-47. doi:10.1037/0022-0663.100.1.30.Problem

Fuchs, L. S., Compton, D. L., Fuchs, D., Paulsen, K., Bryant, J. D., \& Hamlett, C. L. (2005). The Prevention, Identification, and Cognitive Determinants of Math Difficulty. Journal of Educational Psychology, 97(3), 493-513. doi:10.1037/0022-0663.97.3.493

Fuchs, L. S., Fuchs, D., Compton, D. L., Powell, S. R., Seethaler, P. M., Capizzi, A. M., ... Fletcher, J. M. (2006). The Cognitive Correlates of Third-Grade Skill in Arithmetic, Algorithmic Computation, and Arithmetic Word Problems. Journal of Educational Psychology, 98(1), 29-43. doi:10.1037/0022-0663.98.1.29

Ganley, C. M., \& Vasilyeva, M. (2011). Sex differences in the relation between math performance, spatial skills, and attitudes. Journal of Applied Developmental Psychology, 32(4), 235-242. doi:10.1016/j.appdev.2011.04.001 
768 Garner, A., Connor, B., Narad, M., Tamm, L., Simon, J., \& Epstein, J. N. (2014). The Relationship

between ADHD Symptom Dimensions, Clinical Correlates and Functional Impairments. Journal of Developmental and Behavioral Pediatrics, 34(7), 469-477. doi:10.1097/DBP.0b013e3182a39890.The

Gathercole, S. E., \& Pickering, S. J. (2000). Working memory deficits in children with low achievements in the national curriculum at 7 years of age. The British Journal of Educational Psychology, 70(2), 177-94.

Geary, D. C. (1994). Children's mathematical development: Research and practical applications. American Psychological Association.

Geary, D. C., Brown, S. C., \& Samaranayake, V. (1991). Cognitive Addition: A Short Longitudinal Study of Strategy Choice and Speed-of-Processing Differences in Normal and Mathematically Disabled Children. Developmental Psychology, 27(5), 787-797.

Geary, D. C., Hoard, M. K., \& Nugent, L. (2012). Independent contributions of the central executive, intelligence, and in-class attentive behavior to developmental change in the strategies used to solve addition problems. Journal of Experimental Child Psychology, 113(1), 49-65. doi:10.1016/j.jecp.2012.03.003

Gershon, J. (2002). A meta-analytic review of gender differences in ADHD. Journal of Attention Disorders, 5(3), 143-54.

Gold, A. B., Ewing-Cobbs, L., Cirino, P., Fuchs, L. S., Stuebing, K. K., \& Fletcher, J. M. (2013). Cognitive and behavioral attention in children with math difficulties. Child Neuropsychology, 19(4), 420-37. doi:10.1080/09297049.2012.690371

Good, R. H., Kaminski, R. A., Smith, S., Laimon, D., \& Dill, S. (2001). Dynamic indicators of basic early literacy skills (5th ed.). Eugene, OR: University of Oregon. 
791 Gray, S., Rogers, M., Martinussen, R., \& Tannock, R. (n.d.). The inter-relationships between behavioral inattention, working memory and academic fluency in a Canadian community sample of school children.

794 Grills-Taquechel, A. E., Fletcher, J. M., Vaughn, S. R., Denton, C. a, \& Taylor, P. (2013). Anxiety and

795

796

797

798

799

800

801

802

803

804

805

806

807

808

809

810

811

812

813

814 inattention as predictors of achievement in early elementary school children. Anxiety, Stress, and Coping, 26(4), 391-410. doi:10.1080/10615806.2012.691969

Hayes, A. (2013). Introduction to mediation, moderation, and conditional process analysis: A regression-based approach. New York, NY: Guilford Press.

Jacobson, L., Ryan, M., Martin, R. B., Ewen, J., Mostofsky, S. H., Denckla, M. B., \& Mahone, E. M. (2011). Working memory influences processing speed and reading fluency in ADHD. Child Neuropsychology, 17(3), 209-24. doi:10.1080/09297049.2010.532204

Kaufman, S. B. (2007). Sex differences in mental rotation and spatial visualization ability: Can they be accounted for by differences in working memory capacity? Intelligence, 35, 211-223. doi:10.1016/j.intell.2006.07.009

Langberg, J. M., Molina, B. S. G., Arnold, L. E., Epstein, J. N., Altaye, M., Hinshaw, S. P., ... Hechtman, L. (2011). Patterns and predictors of adolescent academic achievement and performance in a sample of children with attention-deficit/hyperactivity disorder. Journal of Clinical Child and Adolescent Psychology, 40(4), 519-31. doi:10.1080/15374416.2011.581620

Lewandowski, L. J., Lovett, B. J., Parolin, R., Gordon, M., \& Codding, R. S. (2007). Extended Time Accommodations and the Mathematics Performance of Students With and Without ADHD Lawrence. Journal of Psychoeducational Assessment, 25(1), 17-28. doi:10.1177/0734282906291961

Li, Y., \& Geary, D. C. (2013). Developmental gains in visuospatial memory predict gains in mathematics achievement. PloS One, 8(7), e70160. doi:10.1371/journal.pone.0070160 
815 Martinussen, R., Grimbos, T., \& Ferrari, J. L. S. (2014). Word-Level Reading Achievement and

816 Behavioral Inattention: Exploring Their Overlap and Relations with Naming Speed and Phonemic 817 Awareness in a Community Sample of Children. Archives of Clinical Neuropsychology,

818 29(September), 680-690. doi:10.1093/arclin/acu040

819 Martinussen, R., Hayden, J., Hogg-Johnson, S., \& Tannock, R. (2005). A meta-analysis of working 820 memory impairments in children with attention-deficit/hyperactivity disorder. Journal of the $821 \quad$ American Academy of Child and Adolescent Psychiatry, 44(4), 377-84.

822 doi:10.1097/01.chi.0000153228.72591.73

823 Martinussen, R., \& Tannock, R. (2006). Working memory impairments in children with attention824 deficit hyperactivity disorder with and without comorbid language learning disorders. Journal of 825 Clinical and Experimental Neuropsychology, 28(7), 1073-94. doi:10.1080/13803390500205700

826 Masters, M. S., \& Sanders, B. (1993). Is the gender difference in mental rotation disappearing?

827 Behavior Genetics, 23(4), 337-341.

828 McKnight, P., McKnight, K., Sidani, S., \& Figueredo, A. (2007). Missing Data: A Gentle Introduction. $829 \quad$ New York, NY: Guilford Press.

830 Miyake, A., Friedman, N. P., Rettinger, D. a., Shah, P., \& Hegarty, M. (2001). How Are Visuospatial 831 Working Memory, Executive Functioning, and Spatial Abilities Related? A Latent-Variable 832 Analysis*1. Journal of Experimental Psychology: General, 130(4), 19. doi:10.1037//0096$833 \quad 3445.130 .4 .621$

834 Modesto-Lowe, V., Yelunina, L., \& Hanjan, K. (2011). Attention-deficit/hyperactivity disorder: a shift 835 toward resilience? Clinical Pediatrics, 50(6), 518-24. doi:10.1177/0009922810394836

836 Passolunghi, M. C., \& Siegel, L. S. (2004). Working memory and access to numerical information in 837 children with disability in mathematics. Journal of Experimental Child Psychology, 88, 348-367. $838 \quad$ doi:10.1016/j.jecp.2004.04.002 
839 Pearce, L., \& Gayle, R. (2009). Oral Reading Fluency as a Predictor of Reading Comprehension With 840 American Indian and White Elementary Students. School Psychology Review, 38(3), 419-427.

841 Petrill, S., Logan, J., Hart, S., Vincent, P., Thompson, L., Kovas, Y., \& Plomin, R. (2012). Math

842 Fluency is Etiologically Distinct From Untimed Math Performance, Decoding Fluency, and 843 Untimed Reading Performance: Evidence From a Twin Study. Journal of Learning Disabilities, 844 45(4), 371-381. doi:10.1177/0022219411407926.Math

845 Pham, A. (2013). Differentiating Behavioral Ratings of Inattention, Impulsivity, and Hyperactivity in 846 Children: Effects on Reading Achievement. Journal of Attention Disorders. doi:10.1177/1087054712473833

848 Pingault, J., Tremblay, R., Vitaro, F., Carbonneau, R., Genolini, C., Falissard, B., \& Côté, S. (2011).

849 Childhood Trajectories of Inattention and Hyperactivity and Prediction of Educational Attainment 850 in Early Adulthood: A 16-Year longitudinal Population-Based Study. American Journal of 851 Psychiatry, 168(11), 1164-1170. doi:10.1176/appi.ajp.2011.10121732

852 Polderman, T. J. C., Boomsma, D. I., Bartels, M., Verhulst, F. C., \& Huizink, a C. (2010). A systematic

858 Preacher, K. J., \& Hayes, A. F. (2008). Asymptotic and resampling strategies for assessing and comparing indirect effects in multiple mediator models. Behavior Research Methods, 40(3), 879-

861 Preacher, K. J., \& Kelley, K. (2011). Effect size measures for mediation models: quantitative strategies 862 for communicating indirect effects. Psychological Methods, 16(2), 93-115. doi:10.1037/a0022658 
863 Qin, S., Cho, S., Chen, T., Rosenberg-Lee, M., Geary, D. C., \& Menon, V. (2014). Hippocampal-

864 neocortical functional reorganization underlies children's cognitive development. Nature

$865 \quad$ Neuroscience, 17(9), 1263-1269. doi:10.1038/nn.3788

866 Rabiner, D., \& Coie, J. (2000). Early Attention Problems and Children's Reading Achievement: A

867 Longitudinal Investigation. Journal of the American Academy of Child \& Adolescent Psychiatry,

868 39(7), 859-867. doi:10.1097/00004583-200007000-00014

869 Raghubar, K., Cirino, P., Barnes, M., Ewing-Cobbs, L., Fletcher, J., \& Fuchs, L. (2009). Errors in

870 multi-digit arithmetic and behavioral inattention in children with math difficulties. Journal of

871 Learning Disabilities, 42(4), 356-71. doi:10.1177/0022219409335211

872 Raghubar, K. P., Barnes, M. a., \& Hecht, S. a. (2010). Working memory and mathematics: A review of

873 developmental, individual difference, and cognitive approaches. Learning and Individual

874 Differences, 20(2), 110-122. doi:10.1016/j.lindif.2009.10.005

875 Ramtekkar, U. P., Reiersen, A. M., Todorov, A. a, \& Todd, R. D. (2010). Sex and age differences in

876 attention-deficit/hyperactivity disorder symptoms and diagnoses: implications for DSM-V and

877 ICD-11. Journal of the American Academy of Child and Adolescent Psychiatry, 49, 217-228.e1-

$878 \quad$ e3. doi:10.1016/j.jaac.2009.11.011

879 Rennie, B., Beebe-Frankenberger, M., \& Swanson, H. L. (2014). A longitudinal study of

880 neuropsychological functioning and academic achievement in children with and without signs of

881 attention-deficit/hyperactivity disorder. Journal of Clinical and Experimental Neuropsychology,

882 36(February 2015), 621-635. doi:10.1080/13803395.2014.921284

883 Roehrig, A. D., Petscher, Y., Nettles, S. M., Hudson, R. F., \& Torgesen, J. K. (2008). Accuracy of the

884 DIBELS oral reading fluency measure for predicting third grade reading comprehension

885 outcomes. Journal of School Psychology, 46(3), 343-66. doi:10.1016/j.jsp.2007.06.006 
886 Rogers, M., Hwang, H., Toplak, M., Weiss, M., \& Tannock, R. (2011). Inattention, working memory, 887 and academic achievement in adolescents referred for attention deficit/hyperactivity disorder (ADHD). Child Neuropsychology, 17(5), 444-58. doi:10.1080/09297049.2010.544648

889

890

891

892

893

894

895

896

897

898

899

900

901

902

903

904

905

906

907

908

909

Rucklidge, J. J., \& Tannock, R. (2002). Neuropsychological profiles of adolescents with ADHD: effects of reading difficulties and gender. Journal of Child Psychology and Psychiatry, 43(8), 988-1003. doi:10.1111/1469-7610.00227

Sheslow, D., \& Adams, W. (2003). Wide Range Assessment of Memory and Learning Second Edition administration and technical manual. Lutz, FL: Psychological Assessment Resources.

Swanson, H. (2011). Working memory, attention, and mathematical problem solving: A longitudinal study of elementary school children. Journal of Educational Psychology, 103(4), 821-837. doi:10.1037/a0025114

Swanson, H., \& Beebe-Frankenberger, M. (2004). The Relationship Between Working Memory and Mathematical Problem Solving in Children at Risk and Not at Risk for Serious Math Difficulties. Journal of Educational Psychology, 96(3), 471-491. doi:10.1037/0022-0663.96.3.471

Swanson, J., Schuck, S., Mann, M., Carlson, C., Hartman, K., \& Sergeant, J. (2001). The SWAN Rating Scale. Retrieved from http://www.adhd.net.

Tannock, R. (2003). Neuropsychology of Attention Disorders. In I. Rapin \& S. Segalowitz (Eds.), Handbook of Neuropsychology (2nd ed., pp. 753-784). Amsterdam: Elsevier.

Thorell, L. B. (2007). Do delay aversion and executive function deficits make distinct contributions to the functional impact of ADHD symptoms? A study of early academic skill deficits. Journal of Child Psychology and Psychiatry, 48(11), 1061-70. doi:10.1111/j.1469-7610.2007.01777.x

Thurber, R. S., Shinn, M. R., \& Smolkowski, K. (2002). What is Measured in Mathematics Tests? Construct Validity of Curriculum-Based Mathematics Measures. School Psychology Review, 31(4), 498-513. 
910 Tighe, E. L., \& Schatschneider, C. (2014). Examining the Relationships of Component Reading Skills 911 to Reading Comprehension in Struggling Adult Readers: A Meta-Analysis. Journal of Learning 912 Disabilities, 43(2), 108-21. doi:10.1177/0022219414555415

913 Trbovich, P. L., \& LeFevre, J.-A. (2003). Phonological and visual working memory in mental addition. $914 \quad$ Memory \& Cognition, 31(5), 738-745. doi:10.3758/BF03196112

915 Wechsler, D. (2003). Wechsler intelligence scale for children-Fourth Edition (WISC-IV). San Antonio, 916 TX: The Psychological Corporation.

917 Willcutt, E. G., Doyle, A. E., Nigg, J. T., Faraone, S. V, \& Pennington, B. F. (2005). Validity of the 918 executive function theory of attention-deficit/hyperactivity disorder: a meta-analytic review. 919 Biological Psychiatry, 57(11), 1336-46. doi:10.1016/j.biopsych.2005.02.006

920 Willcutt, E. G., Pennington, B. F., Olson, R. K., \& DeFries, J. C. (2007). Understanding comorbidity: a 921 twin study of reading disability and attention-deficit/hyperactivity disorder. American Journal of $922 \quad$ Medical Genetics., 144B(6), 709-14. doi:10.1002/ajmg.b.30310

923 Woodcock, R. W., McGrew, K. S., \& Mather, N. (2001). Woodcock-Johnson III. Itasca, IL: Riverside 924 Publishing.

925 Young, D. J., Levy, F., Martin, N. C., \& Hay, D. A. (2009). Attention deficit hyperactivity disorder: a 926 Rasch analysis of the SWAN Rating Scale. Child Psychiatry and Human Development, 40(4), 927 543-59. doi:10.1007/s10578-009-0143-z 


\section{Table $\mathbf{1}$ (on next page)}

Correlations table

Table 1

Partial Correlations, Controlling for Age, Between Study Variables for Full Sample $(N=204)$ 
Table 1

Partial Correlations, Controlling for Age, Between Study Variables for Full Sample $(N=204)$

\begin{tabular}{llllllllll}
\hline Variables & 1 & 2 & 3 & 4 & 5 & 6 & 7 & 8 & 9
\end{tabular}

1. Teacher-rated Inattention

2. Word Reading

$-.49 * *-$

3. Reading Fluency

$-.54 * * \quad .81 * * \quad-$

4. Math Calculation

$\begin{array}{llll}-.48 * * & .49 * * & .51 * * & -\end{array}$

5. Addition Fluency

$\begin{array}{llll}-.48 * * & .42 * * & .52 * * & .59 * *\end{array}$

6. Subtraction Fluency

$-.40 * * \quad .44 * *$

$.47 * *$

$.58 * *$

$.82 * *$

7. Auditory-Verbal WM

$-.23 * * \quad .31 * *$

$.35 * *$

$.33 * *$

$.30 * *$

$.34 * *$

8. Visual-Spatial WM

$-.34 *$

$.24 * *$

$.24 * *$

$.33 * *$

$.33 * *$

$.32 * *$

$.14 \quad-$

8. Sex

$-.37 * * \quad .08$

$.16^{*} \quad .08$

.00

$\begin{array}{lll}-.15^{* *} & -.05 & .16^{*}\end{array}$

8. Parent education level

$-.22 * * \quad .23 *$

$.31 * *$

$.22 * *$

$.20 * *$

$.24 * *$

$.16^{*} \quad .10 \quad .03$

\section{Significant correlation: $* p<.05 . * * p<.01$.}

3 Measured at Year 1 Time A: Teacher-rated Inattention, Age, Sex, Parent education level.

4 Measured at Year 1 Time B: All working memory measures.

5 Measured at Year 2 Time B: All academic measures.

6 Teacher-rated Inattention, Addition and Subtraction Fluency: total raw scores. Math Calculation,

7 Reading Fluency, Word Reading, working memory variables: Standard Scores.

8

9

10

11

12

13

14

15

16

17

18

19

20 


\section{Table 2 (on next page)}

Means and Standard Deviations for Study Variables, for Girls $(n=101)$ and Boys ( $n=$ 103)

Table 2 [i]

Means and Standard Deviations for Study Variables, for Girls $(n=101)$ and Boys $(n=103)[i]$ 
Table 2

Means and Standard Deviations for Study Variables, for Girls $(n=101)$ and Boys $(n=103)$

Total M (SD) Males M (SD) Females M (SD) $\quad F^{\text {a }}$

\begin{tabular}{lcccc}
\hline Teacher-rated Inattention & $-1.58(13.92)$ & $3.43(13.07)$ & $-6.69(12.91)$ & $30.95^{* *}$ \\
Word Reading & $102.0(11.79)$ & $100.53(12.52)$ & $103.56(10.84)$ & 3.41 \\
Reading Fluency & $102.2(44.26)$ & $95.95(44.83)$ & $108.47(42.98)$ & $4.14^{*}$ \\
Math Calculation & $95.00(9.61)$ & $94.15(9.35)$ & $95.87(9.83)$ & 1.65 \\
Addition Fluency & $41.67(18.14)$ & $42.37(19.51)$ & $40.96(16.70)$ & 0.31 \\
Subtraction Fluency & $29.82(13.90)$ & $32.23(14.88)$ & $27.36(12.43)$ & $6.44^{*}$ \\
Auditory-Verbal WM & $8.77(2.45)$ & $8.78(2.33)$ & $8.76(2.58)$ & 0.002 \\
Visual-Spatial WM & $8.12(3.25)$ & $7.73(3.31)$ & $8.51(3.16)$ & 3.01
\end{tabular}

$* p>.05, * * p>.001$

${ }^{\text {a }} \mathrm{F}$ statistic for one-way ANOVA

Measured at Year 1 Time A: Teacher-rated Inattention, Age, Sex, Parent education level.

Measured at Year 1 Time B: All working memory measures.

Measured at Year 2 Time B: All academic measures.

Teacher-rated Inattention, Addition and Subtraction Fluency: total raw scores. Math Calculation, Reading Fluency, Word Reading, working memory variables: Standard Scores. 
Figure $\mathbf{1}_{\text {(on next page) }}$

Significant moderated mediation: Visual-spatial WM as a mediator of the relationship between teacher-rated inattention and boys' math addition scores one year later.

Figure 1. Significant moderated mediation: Visual-spatial WM as a mediator of the relationship between teacher-rated inattention and boys' math addition scores one year later. 


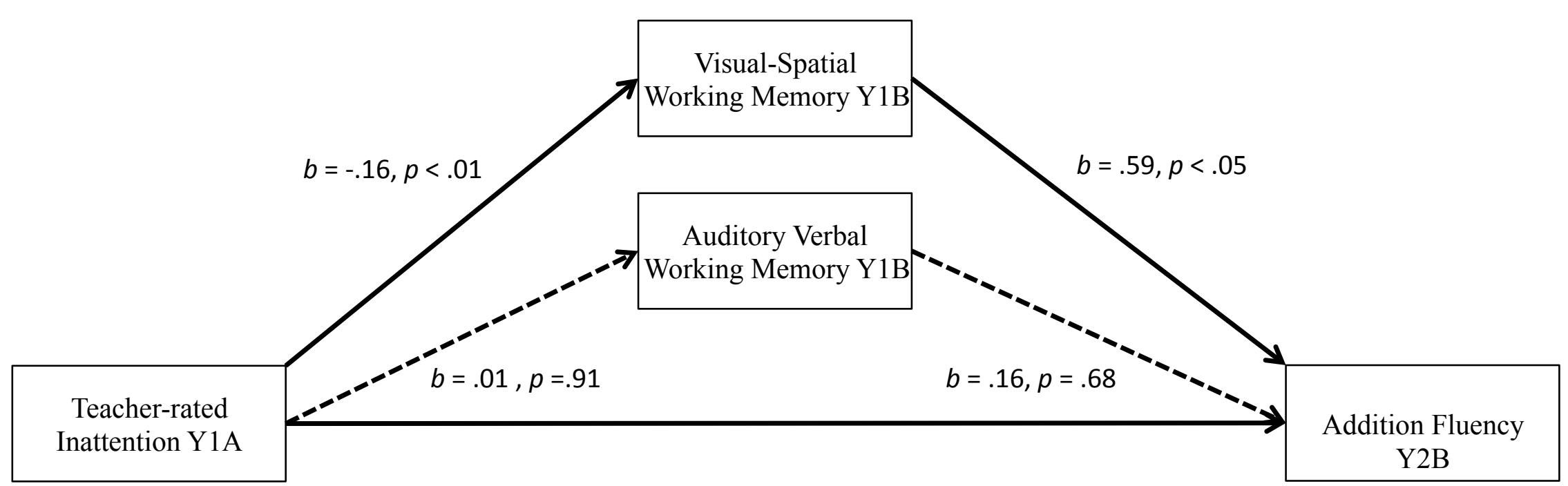

Direct effect, $b=-0.28$, CI $[-0.43,-0.14]$

Conditional indirect effect of visual-spatial WM for boys, $b=-0.06$ CI $[-0.13,-0.01]$ 
Figure 2 (on next page)

Figure 2. Significant moderated mediation: Visual-spatial WM as a mediator of the relationship between teacher-rated inattention and boys' math subtraction scores one year later.

Figure 2. Significant moderated mediation: Visual-spatial WM as a mediator of the relationship between teacher-rated inattention and boys' math subtraction scores one year later. 


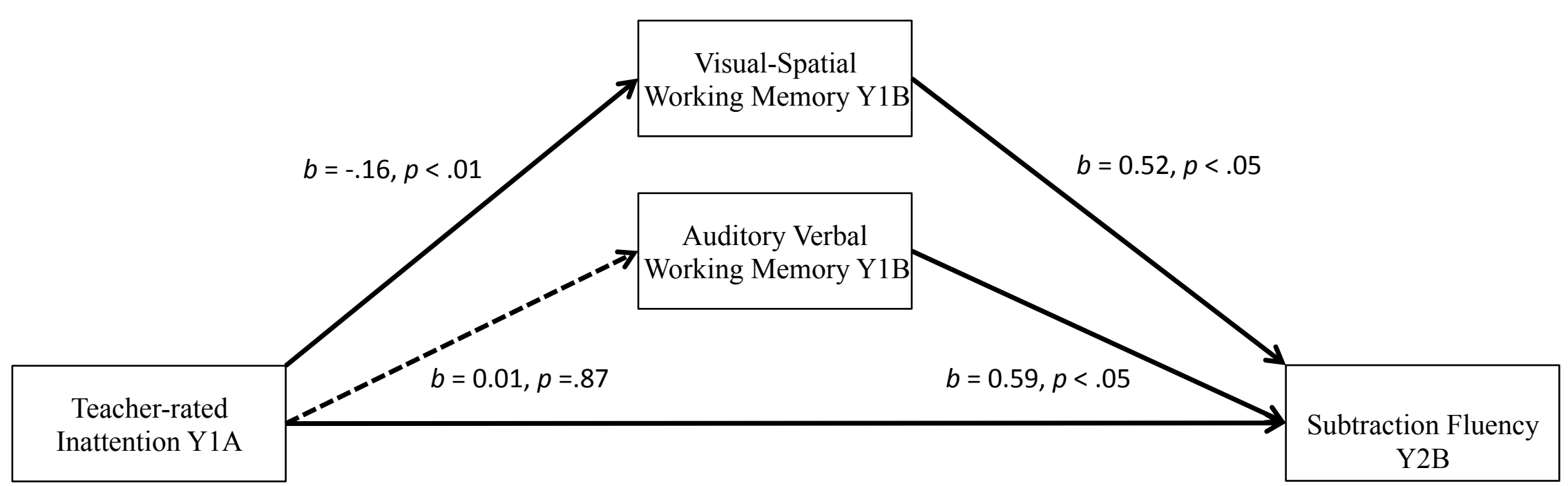

Direct effect, $b=-0.13$, CI $[-0.24,-0.02]$

Conditional indirect effect of visual-spatial WM for boys, $\mathrm{b}=-0.05 \mathrm{CI}[-0.11,-.01]$ 
Figure $\mathbf{3}$ (on next page)

Significant moderated mediation: Visual-spatial WM as a mediator of the relationship between teacher-rated inattention and boys' math calculation scores one year later.

Figure 3. Significant moderated mediation: Visual-spatial WM as a mediator of the relationship between teacher-rated inattention and boys' math calculation scores one year later. 


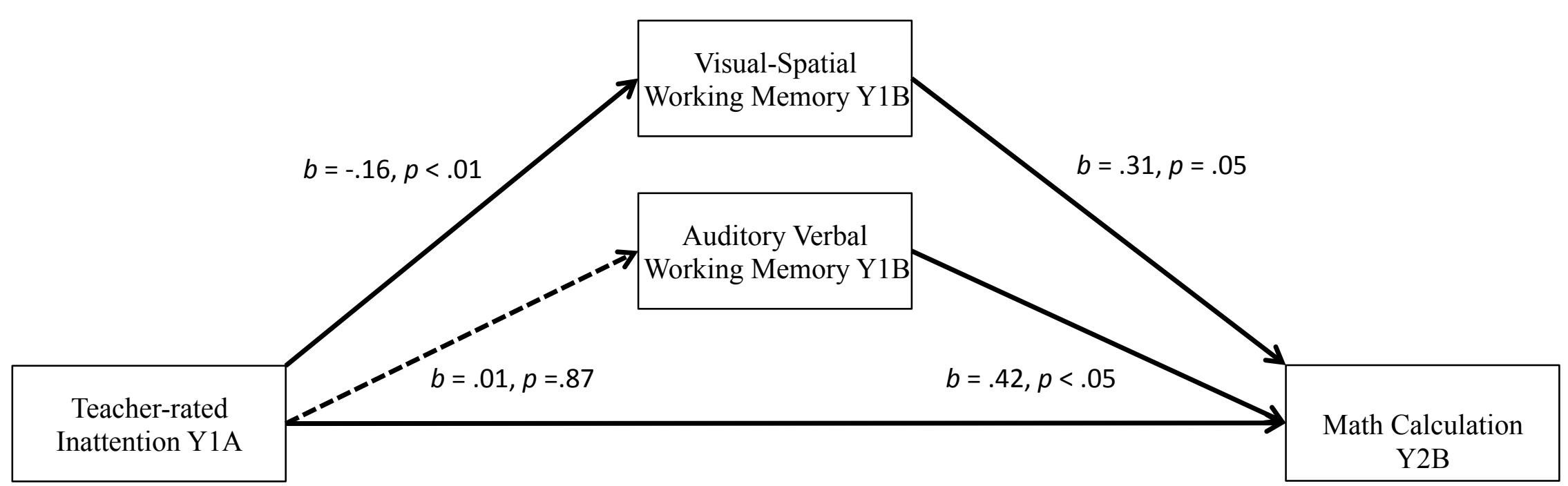

Direct effect, $b=-0.14$, CI $[-0.22,-0.06]$

Conditional indirect effect of visual-spatial WM for boys, $b=-0.03 \mathrm{CI}[-0.07,-0.00]$ 